\title{
Anharmonicity but absence of surface melting on $\mathrm{Al}(001)$
}

\author{
A. M. Molenbroek and J. W. M. Frenken \\ FOM Institute for Atomic and Molecular Physics, Kruislaan 407, 1098 SJ Amsterdam, The Netherlands
}

(Received 23 August 1993)

\begin{abstract}
Medium-energy ion-scattering measurements and Monte Carlo simulations have been used to study the $\mathrm{Al}(001)$ surface as a function of temperature. Surface melting does not occur and the surface stays well ordered up to the bulk melting point $T_{m}=933.52 \mathrm{~K}$. The combination of the effective-medium potential, used to simulate the interacting $\mathrm{Al}$ atoms, and ion-scattering calculations on the simulated $\mathrm{Al}(001)$ surface, reproduces the ion-scattering intensities very well. The Monte Carlo simulations show that the surface relaxations and the vibration amplitudes both display strongly anharmonic behavior which extends several layers into the crystal and increases with increasing temperature.
\end{abstract}

\section{INTRODUCTION}

The temperature-dependent behavior of single-crystal surfaces has received increasing interest in the past decade. Several disordering processes and related phenomena, such as roughening, 1,2 surface melting, ${ }^{3,4}$ faceting, ${ }^{5,6}$ and deconstruction ${ }^{7-9}$ have been identified on crystal surfaces. Especially surface melting, the appearance of a thin liquidlike layer on top of a solid surface below the bulk melting point, has been studied extensively by means of experiments as well as theory ${ }^{10-13}$ and computer simulations. ${ }^{14-17}$ The open fcc(110) faces of, e.g., $\mathrm{Al},{ }^{16,18-22} \mathrm{Au},{ }^{23-25} \mathrm{Cu},{ }^{26,27}$ and $\mathrm{Pb}$ (Refs. 3 and 28-30), have been shown to exhibit surface melting while the close-packed (111) faces of these materials are stable up to the bulk melting point. ${ }^{20,23,26,27,29,31}$

Thermodynamically, one can easily predict the surface melting behavior by means of a comparison between the orientation-dependent free energies $\gamma$ for the solid-vapor (sv), solid-liquid (sl), and liquid-vapor (lv) interfaces. ${ }^{32}$ Surface melting is expected when

$$
\Delta \gamma \equiv \gamma_{\mathrm{sv}}-\left(\gamma_{\mathrm{s} 1}+\gamma_{\mathrm{lv}}\right)>0
$$

and surface stability when $\Delta \gamma<0$. In practice it is difficult to predict surface melting and nonmelting on the basis of the sign of $\Delta \gamma$, because accurate values for $\gamma_{\mathrm{sv}}$ and $\gamma_{\mathrm{sl}}$ are usually not available. Not only surface melting or stability can occur, but also incomplete melting or layering is possible for surfaces with a relatively small absolute value of $\Delta \gamma \cdot{ }^{33}$ In addition, some ranges of orientations can break up into melted and dry facets at elevated temperatures. $5,34,35$

Energetically, the (001) surfaces of fcc metals are intermediate between the melting (110) and the nonmelting (111) surfaces. Disordered first layers as well as stable surfaces have been reported close to the bulk melting point. The $\mathrm{Pb}(001)$ surface has been studied by mediumenergy ion scattering (MEIS) (Refs. 29 and 35) and high resolution low-energy electron diffraction (LEED). ${ }^{36}$ With increasing temperature the surface expands anomalously followed by the creation of a high density of sur- face vacancies. It develops a finite amount (approximately one monolayer) of disorder close to $T_{m}$, probably caused by layering effects.

The $\mathrm{Cu}(001)$ surface also does not show (complete) surface melting, according to a light-emission study, employing the difference in infrared emissivity of solid and liquid Cu. ${ }^{26}$ Circular areas around the (001) and (111) poles remain dark close to the bulk melting point, while all other orientations exhibit an increase in emissivity. A recent molecular-dynamics simulation of low-index faces of $\mathrm{Cu}$ indicates that the (001) face develops a limited amount of disorder close to the bulk melting point. ${ }^{27}$

The purpose of the present work is to investigate the high-temperature behavior of $\mathrm{Al}(001)$. This surface has been studied before by angle-resolved photoemission. ${ }^{37,38}$ In that study, a decrease in the intensity of the surface state above $700 \mathrm{~K}$ was observed. The observation that some residual intensity was still present at $0.3 \mathrm{~K}$ below the bulk melting point was interpreted as evidence against surface melting of $\mathrm{Al}(001)$.

We have studied the structure and vibrations of $\mathrm{Al}(001)$ as a function of temperature by means of MEIS. This technique has been shown to be very sensitive in detecting disordered or molten surface layers. ${ }^{39}$ The ion-scattering results can be interpreted either in terms of strongly anharmonic surface vibrations or as the signature of a limited amount of surface disorder at $T_{m}$. In addition, we have performed Monte Carlo (MC) simulations of the $\mathrm{Al}(001)$ surface, using the effective-medium theory (EMT) to describe the Al interactions, and subsequently we have computed the ion-scattering yield from the simulated surface. The simulated $\mathrm{Al}(001)$ surface remains well ordered up to $T_{m}$ and exhibits clear signs of anharmonicity. The ion-scattering yields calculated for the simulated surface are in quantitative agreement with the experimental results.

The employed EMT potential is the same as that used previously in simulations of $\mathrm{Al}(110)$ and $\mathrm{Al}(111){ }^{21}$ For both the melting $\mathrm{Al}(110)$ and the nonmelting $\mathrm{Al}(111)$ surfaces, the EMT-simulations together with ion-scattering calculations have led to accurate predictions of the ionscattering intensities and their temperature dependence. 


\section{EXPERIMENT}

\section{A. Sample preparation}

The specimen was spark cut to a rectangular shape (dimensions are $10 \times 10 \times 3 \mathrm{~mm}^{2}$ ) from a single-crystal high-purity Al bar. For mounting purposes a $0.5 \times 0.3$ $\mathrm{mm}^{3}$ groove was spark cut along the edges of the crystal. The crystal was etched in a solution of sulfuric acid (96\%), phosphoric acid (85\%), and nitric acid (65\%) in a ratio $(5: 14: 1)$, heated to $85^{\circ} \mathrm{C}$. It was oriented by Laue backdiffraction to within $0.1^{\circ}$ of the [001] axis and mechanically polished subsequently with $6-, 3-$ and $1-\mu \mathrm{m}$ diamond paste followed by a $0.05-\mu \mathrm{m}$ aluminum-oxide suspension in water. The crystal was mounted in a Mo container using Mo clamps and transferred into the UHV MEIS system, described previously ${ }^{40-42}$ (base pressure $8 \times 10^{-9} \mathrm{~Pa}$ ). The crystal was initially treated in situ by means of the following procedure: $2 \mathrm{~h}$ sputtering with $700 \mathrm{eV} \mathrm{Ar}^{+}$ions $\left(3.5 \mu \mathrm{A} / \mathrm{cm}^{2}\right)$ followed by $40 \mathrm{~h}$ annealing up to $850 \mathrm{~K}$. After this, MEIS and Auger-electron spectroscopy (AES) showed only $\mathrm{C}$ and $\mathrm{O}$ contamination.

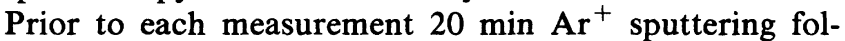
lowed by $30 \mathrm{~min}$ annealing up to $800 \mathrm{~K}$ were sufficient to produce a clean surface. This was checked by means of AES, which did not show $\mathrm{C}$ or $\mathrm{O}$ signals. The upper estimate of the peak ratios of $\mathrm{Al}(68 \mathrm{eV}) / \mathrm{C}(273 \mathrm{eV})$ and $\mathrm{Al}$ $(68 \mathrm{eV}) / \mathrm{O}(503 \mathrm{eV})$ in the derivative spectrum was 200 , based on statistical counting noise at 273 and $503 \mathrm{eV}$. Also at temperatures close to the bulk melting point no impurities could be detected (the typical detection limit is 0.01 monolayer). Low-energy electron diffraction (LEED) showed a sharp $(1 \times 1)$ pattern with a low background intensity.

\section{B. Temperature control}

The Mo container with the crystal was heated radiatively and by means of electron bombardment. Two Ta radiation shields reduced heating of the surroundings of the sample. The temperature was measured with a Pt100 resistor (inside the Mo container at the reverse side of the crystal) and also monitored by an infrared (2.0-2.6 $\mu \mathrm{m})$ pyrometer at the surface side. The absolute emissivity of the surface varied $\pm 5 \%$ over the surface, because of microscopic roughness caused by the in situ cleaning treatment. LEED, AES, and MEIS did not show variations in surface quality over the specimen.

Close to the melting point, we did not observe sudden changes in the infrared emissivity of the surface, in contrast with observations on $\mathrm{Pb}$ surfaces. ${ }^{3,35}$ The bulk melting point, $T_{m}=933.52 \mathrm{~K},{ }^{43}$ of the Al crystal was determined by partially melting the crystal. Lateral temperature differences over the sample surface were estimated to be less than $0.01 \mathrm{~K}$.

The vapor pressure of $\mathrm{Al}$ at the bulk melting point is so low $\left[4 \times 10^{-7} \mathrm{~Pa}\right.$ (Ref. 44)] that the evaporation of $\mathrm{Al}$ atoms can be neglected ( $\approx$ two monolayers per hour). Thus, the crystal can be considered to be in thermodynamic equilibrium with its own vapor at all experimental temperatures.

\section{Medium-energy ion scattering}

The ion-scattering measurements were performed in the (100) scattering plane which is shown in the inset of Fig. 1. A 100-keV parallel proton beam was aligned with the $[01 \overline{1}]$ direction. Backscattered protons were detected over a range of $20^{\circ}$ around the [011] direction. The scattering chamber and the detection system have been described previously. ${ }^{40-42,45}$ The shadowing and blocking effects in such a doubly aligned geometry result in a surface peak in the energy spectra of backscattered ions and a low minimum yield at lower energies. The area of the surface peak in the energy spectrum, for a $1.2^{\circ}$ angular range around [011], was converted into the number of Al monolayers visible to the ion beam and the detector [one monolayer $\mathrm{Al}(001)$ contains $1.22 \times 10^{15} \mathrm{Al}$ atoms $/ \mathrm{cm}^{2}$ ]. The details of this conversion have been described elsewhere. ${ }^{46,47}$ An ordered crystalline surface gives rise to a small surface peak. A disordered or molten surface film on top of an ordered substrate gives an extra contribution to the backscattered signal, which corresponds to the number of disordered monolayers.

Energy spectra of backscattered ions have been measured at temperatures between room temperature and the bulk melting point. In Fig. 1 the resulting number of visible $\mathrm{Al}(001)$ monolayers is shown as a function of temperature. A gradual increase up to the bulk melting point is observed. The Al yield does not diverge at $T_{m}$, so this surface does not exhibit (complete) surface melting. Furthermore, at temperatures close to the bulk melting point the surface peak does not reach the so-called "random height," indicating that there is no (thick) disordered film at the surface.

We have performed ion-scattering calculations ${ }^{48}$ for a nonrelaxed $\mathrm{Al}(001)$ surface. In these calculations, the vibrations were chosen isotropic and uncorrelated. At room temperature we can fit the experimental scattering

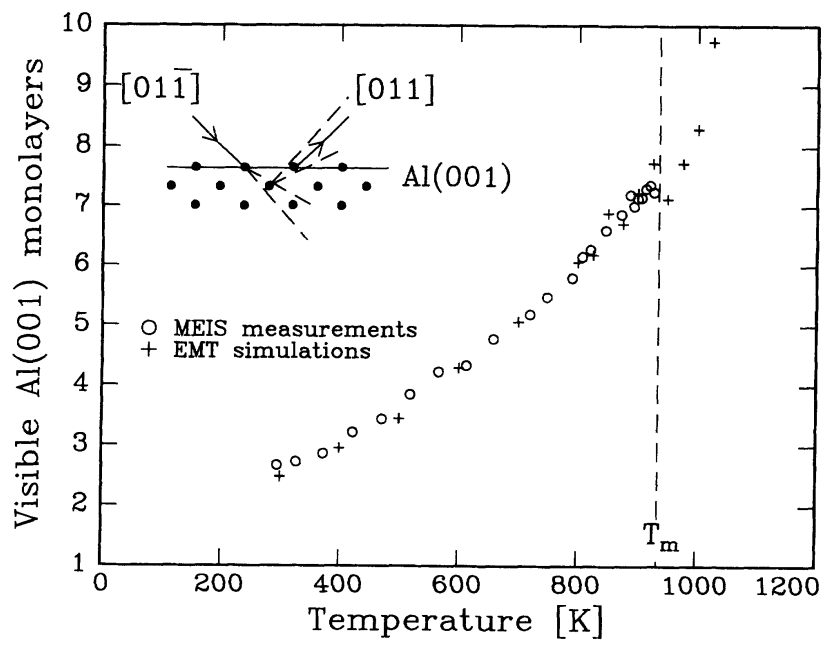

FIG. 1. Number of visible $\mathrm{Al}(001)$ monolayers for $100-\mathrm{keV}$ $\mathrm{H}^{+}$with incoming direction [011] ] and outgoing direction [011]. The (100) scattering plane and the scattering geometry are shown in the inset. Measurements and simulations are discussed in the text. 
yield by giving the first- and second-layer atoms vibration amplitudes enhanced by $60 \%$ and $25 \%$ with respect to the bulk vibration amplitude $\left(\sigma_{b}=0.104 \AA\right)$. With the same enhancement factors for the amplitudes at temperatures close to the bulk melting point, the calculations underestimate the experimental yield at $T_{m}$ by about one monolayer. This additional monolayer is either due to disorder or surface anharmonicity. If we assume that the extra yield is due to anharmonic vibrations, we have to increase the vibration amplitudes of the top two layers with an extra $50 \%$ to fit the data.

To interpret the measurements in more detail and to test the assumption of anharmonic vibrations, we have simulated the $\mathrm{Al}(001)$ surface using the Monte Carlo method and the effective-medium theory to describe the interactions between the atoms. The comparison between experiments and simulations is made quantitative by use of ion-scattering calculations on the simulated surfaces for the same scattering conditions as used in the experiments.

\section{MONTE CARLO SIMULATIONS}

\section{A. Effective-medium theory}

We have used the effective-medium theory (EMT) (Ref. 49) to calculate the total energy of a system of interacting $\mathrm{Al}$ atoms. In the EMT the potential energy of an atom is a function of the average electron density it experiences due to the surrounding atoms, and the total potential energy $E_{\text {tot }}$ of the system is obtained by summing the atomic contributions. The details of the potential we have used have been given in Ref. 21 .

Prior to the slab calculations of the (001) surface, the bulk lattice constant for the employed potential was determined at various temperatures. For this purpose, a unit cell of $256 \mathrm{Al}$ atoms, vibrating around their fcc lattice positions, with three-dimensional periodic boundary conditions, was equilibrated at constant temperature $T$ and zero external pressure. The equilibration was performed by moving the atoms in the cell according to the Metropolis Monte Carlo method. ${ }^{50}$ To make the calculation computationally efficient without throwing away essential interactions, we have cut off the active radius of the potential between the fourth and fifth nearestneighbor shells. This is close to the cell size but because the interaction energy is very small at these large distances this will hardly affect the simulations. The unit cell was considered to be in equilibrium when the total potential energy and the total volume did not show other than statistical fluctuations. From a fit to the equilibrium volume we obtained the lattice constant $a(T)$, between $300 \mathrm{~K}$ and the bulk melting point, as the polynomial: ${ }^{51}$

$a(T)=4.018+7.755 \times 10^{-5} T+2.158 \times 10^{-8} T^{2} \AA$.

The corresponding bulk coefficient of linear thermal expansion, given by

$$
\alpha(T)=\frac{1}{a(T)} \frac{d a(T)}{d T},
$$

increases from $\alpha=2.23 \times 10^{-5} \mathrm{~K}^{-1}$ at $300 \mathrm{~K}$ to
$2.87 \times 10^{-5} \mathrm{~K}^{-1}$ at $T_{m}$. The value of the thermalexpansion coefficient at room temperature is close to the experimental value of $\alpha=2.35 \times 10^{-5} \mathrm{~K}^{-1} .{ }^{52}$ So the EMT potential gives a good description of the bulk anharmonicity of $\mathrm{Al}$. The bulk cohesive energy at $0 \mathrm{~K}$ is $-3.28 \mathrm{eV} /$ atom which is close to the experimental value of $-3.34 \mathrm{eV} /$ atom. ${ }^{53}$

The slab calculations of the surface were performed for a rectangular box of 30 (001) layers of $5 \times 5$ atoms per layer. The upper 20 layers were allowed to move, the lower 10 were fixed to mimic the underlying bulk of the crystal. The influence of the fixing of the layers on the free layers extends over approximately five layers, which we verified by increasing the number of free layers. The atoms in the starting cell at $300 \mathrm{~K}$ were placed on fcc lattice positions with the corresponding lattice constant. Periodic boundary conditions were applied parallel to the surface plane. Within about $10^{4} \mathrm{MC}$ cycles the cell was equilibrated. One MC cycle consists of $N_{\text {free }}$ trial moves of a free atom in a randomly chosen direction, where $N_{\text {free }}$ is the number of free atoms in the cell. The free atoms are chosen in a random order. The magnitudes of the attempted random displacements were adjusted to yield an acceptance probability of $50 \%$. The criterion for equilibration of the unit cell was that the total cohesive energy and some layer-dependent averaged quantities, such as vibration amplitudes and structure factors, merely showed statistical fluctuations. We checked for finitesize effects of the small cell by increasing the number of atoms per layer as well as the number of free layers. A cell size of $5 \times 5 \times 20$ atoms was sufficient for reliable values of the cohesive energy and the averaged quantities.

The equilibration of the cell at higher temperatures was usually started by expanding a cell, equilibrated at a lower temperature, to the appropriate lattice constant. After equilibration, a few thousand MC cycles were used to determine equilibrium averages and to produce a set of mutually uncorrelated snapshots that were used as input for the ion-scattering calculations (Sec. III C).

\section{B. EMT results}

From the profile of the average density along the surface normal ( $z$ direction) it is clear that the simulated $\mathrm{Al}(001)$ surface remains well ordered up to $T \approx 1000 \mathrm{~K}$ (Fig. 2). This can also be seen from the snapshots of the atomic positions in the calculated unit cell in Fig. 3. Between 1000 and $1050 \mathrm{~K}$ the unit cell loses its order, starting from the surface.

The density profiles parallel to the surface also show an ordered surface up to $1000 \mathrm{~K}$, as illustrated by the crosses in Fig. 4 (only position distributions of two atoms in the first layer have been shown). The solid curves are Gaussian functions $f(x)$ to these profiles:

$$
f(x)=\frac{N}{\sqrt{2 \pi \sigma^{2}}} \sum_{i=-\infty}^{\infty} \exp \left[-\frac{(x-b i)^{2}}{2 \sigma^{2}}\right] .
$$

Here, $\sigma^{2}$ is the mean-square displacement in the $x=[110]$ direction of atoms in the first layer, which was obtained from the calculated structure factor (see below), 


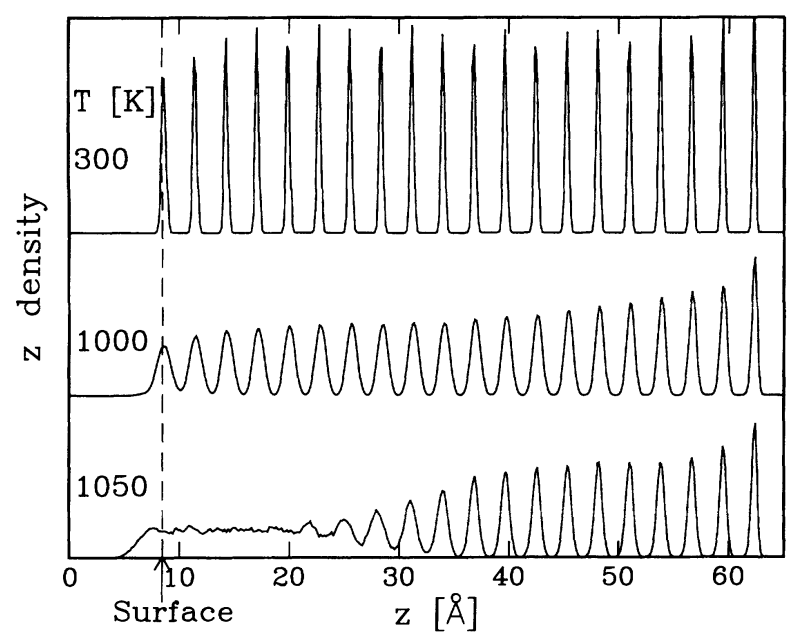

FIG. 2. Density profiles perpendicular to the surface at $T=300,1000$, and $1050 \mathrm{~K}$. Only the free atom layers are shown.

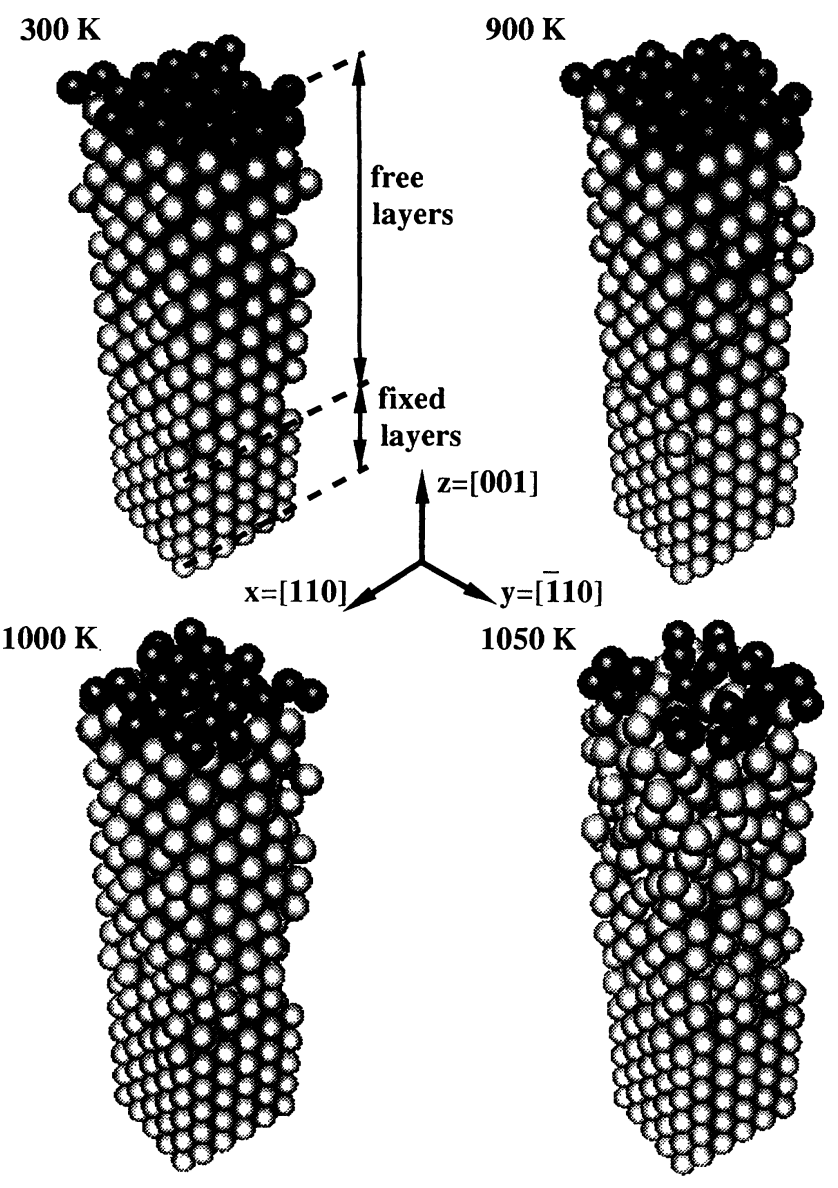

FIG. 3. Perspective view of snapshots of the unit cell at $T=300,900,1000$, and $1050 \mathrm{~K}$. The first-layer atoms have been shaded darker.

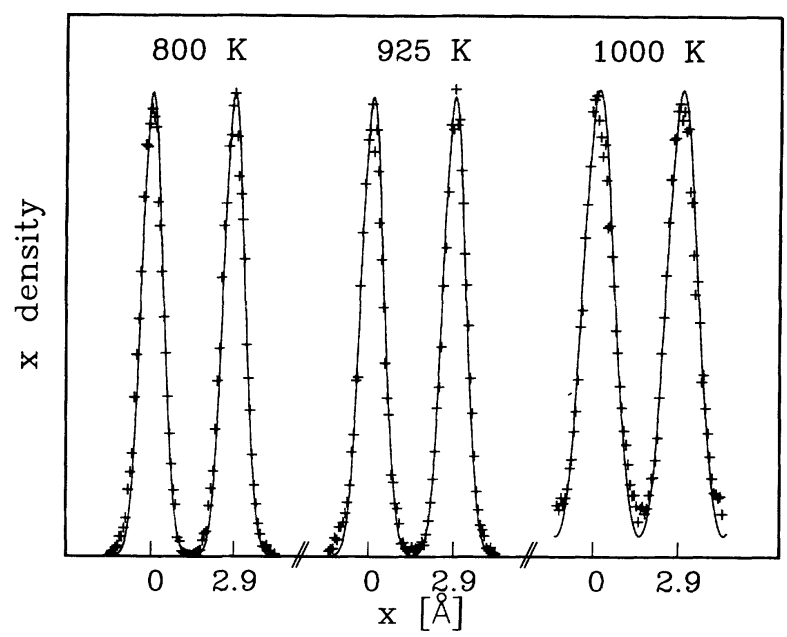

FIG. 4. Density profiles parallel to the surface $(x=[110]$ direction) of the first-layer atoms at $T=800,925$, and $1000 \mathrm{~K}$. Position distributions of only two atoms are shown; the heights are scaled to the same value for the three different temperatures. The curves are Gaussian fits to the EMT results (see text).

the peak distance $b$ is $\frac{1}{2} \sqrt{2} a(T)$ and $N$ is the number of Monte Carlo cycles performed. The distributions along the $x=[110]$ and $y=[110]$ directions are identical because of symmetry. At low temperatures, up to about $800 \mathrm{~K}$, the distributions are purely Gaussian for all layers. At higher temperatures, however, the simulated probability density in between surface lattice sites is seen to be significantly higher than the Gaussian density expected for harmonically vibrating atoms.

The $z$-density profile at $1050 \mathrm{~K}$ in Fig. 2 and the snapshot at that temperature in Fig. 3 do not correspond to an equilibrium situation. Per extra $10^{4} \mathrm{MC}$ cycles approximately one additional layer is molten. Of course, the unit cell will never melt completely because of the order induced by the fixed bulk layers. If this surface, after the melting of approximately eight layers at $1050 \mathrm{~K}$, is cooled down to $1000 \mathrm{~K}$, it orders again in about $5 \times 10^{4}$ MC cycles. The melting point of aluminum in these simulations is, on the basis of these results: $T_{m}=1035 \pm 10 \mathrm{~K}$, which is higher than the experimental melting point. The origin of the difference might be that the liquid is not described as accurately as the solid by the effective-medium potential, which has been optimized for the perfect fcc structure. ${ }^{49}$ In spite of the incorrect simulation value for $T_{m}$, we compare the MC simulations with the experiments on an absolute rather than a relative temperature scale. For the $\mathrm{Al}(001)$ surface, this is justified because there is no melting of this surface below $T_{m} \cdot{ }^{54}$

From the $z$-density profiles, the relaxations $\Delta_{i, i+1}=d_{i, i+1} / d_{\text {bulk }}$ of the surface layers were determined. The distance $d_{i, i+1}$ between the neighboring layers $i$ and $i+1$ was calculated by taking the difference between the centers of mass of the corresponding two neighboring peaks in the $z$-density profile. $d_{\text {bulk }}$ was chosen as the average over $d_{10,11} \ldots d_{14,15}$ and found to 
TABLE I. Experimental values and predictions for the first-layer relaxation of $\mathrm{Al}(001)$.

\begin{tabular}{lclc}
\hline \multicolumn{1}{c}{ Reference } & $\Delta_{1,2}[\%]$ & \multicolumn{1}{c}{ Method } & Temperature $(\mathrm{K})$ \\
\hline 55 & 0 & low-energy electron diffraction & 300 \\
56 & 0 & low-energy electron diffraction & 300 \\
57 & $0 \pm 2$ & x-ray-absorption fine structure & 300 \\
This work & $0 \pm 5$ & medium-energy ion scattering & $300-933$ \\
58 & -1.5 & medium-energy electron diffraction & 77 \\
49 & -3.0 & EMT & 0 \\
This work (Ref. 59) & $-0.9 \pm 0.2$ & EMT & 0 \\
60 & -4.90 & dipole-layer, Hartree, band-structure model & 0 \\
61 & -5.0 & corrected EMT & 0 \\
62 & 0.0 & embedded atom method & 0 \\
63 & $+1.2 \pm 0.4$ & first-principles calculation & 0 \\
64 & +0.7 & semiempirical quantum chemical model & 0 \\
\hline \hline
\end{tabular}

be equal to $a(T) / 2$, where $a(T)$ is given by Eq. (2). Table I gives a summary of experimental and calculated values for the first-layer relaxation of $\mathrm{Al}(001)$.

In the scattering geometry used, MEIS is not very sensitive to surface relaxations. Relaxations of a few percent will not result in a significant shift of the blocking minimum with respect to the bulk axis.

Figure 5 reveals that the simulated surface relaxations are strongly temperature dependent. This directly reflects the anharmonicity of the surface layers. All surface layers relax outward with increasing temperature and at the experimental bulk melting point the firstinterlayer distance is expanded by $2.5 \%$. This corresponds to an enhancement in the thermal-expansion coefficient of the first-interlayer distance, from room temperature to the bulk melting point, by a factor 2.5 with respect to the bulk thermal-expansion coefficient.

For $\mathrm{Al}(110)$ the onset of surface melting is correlated with the thermal generation of high densities of adatoms and vacancies. These adatom/vacancy pairs are thought to be a precursor of surface melting. ${ }^{21}$ In Fig. 6 the occupation of the first layer and the density of adatoms are shown as a function of temperature for $\mathrm{Al}(001)$ and, for

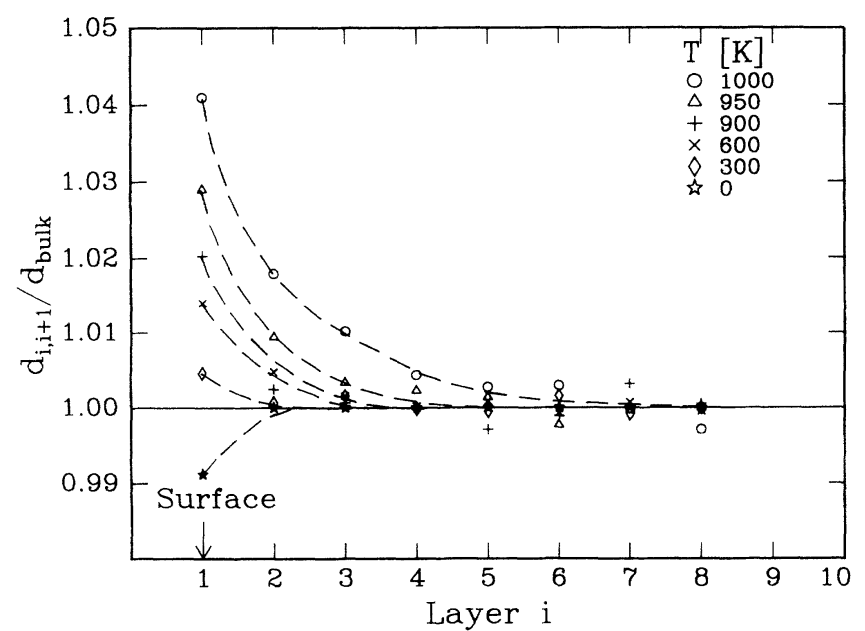

FIG. 5. Relaxation of the first eight layers at $T=0,300,600$, 900,950 , and $1000 \mathrm{~K}$. comparison, for $\mathrm{Al}(110)$. At the $\mathrm{Al}(001)$ surface the formation of adatom/vacancy pairs starts only at $T=1025$ $K$, well above the experimental melting point of this surface. The very small density of adatoms present below the melting point is mainly caused by the way of counting: an atom is counted as an adatom if its $z$ coordinate is more than half an interlayer distance above the first layer. Because of the large vibration amplitudes at high temperatures, atoms in the first layer are sometimes mistaken for adatoms. Compared to the $\mathrm{Cu}(001)$ surface, which has been studied with molecular dynamics using EMT, ${ }^{27}$ and which only develops a limited amount of surface disorder, the number of adatoms on the $\mathrm{Al}(001)$ surface close to the melting point is an order of magnitude lower.

In order to investigate the order parallel to the surface, we calculated the in-plane layer-dependent structure factor

$$
S_{j}(\mathbf{k})=\frac{1}{N_{j}} \sum_{l=1}^{N_{j}} e^{i \mathbf{k} \cdot \mathbf{r}_{l}} .
$$

Here $N_{j}$ is the actual number of atoms in layer $j$ for the

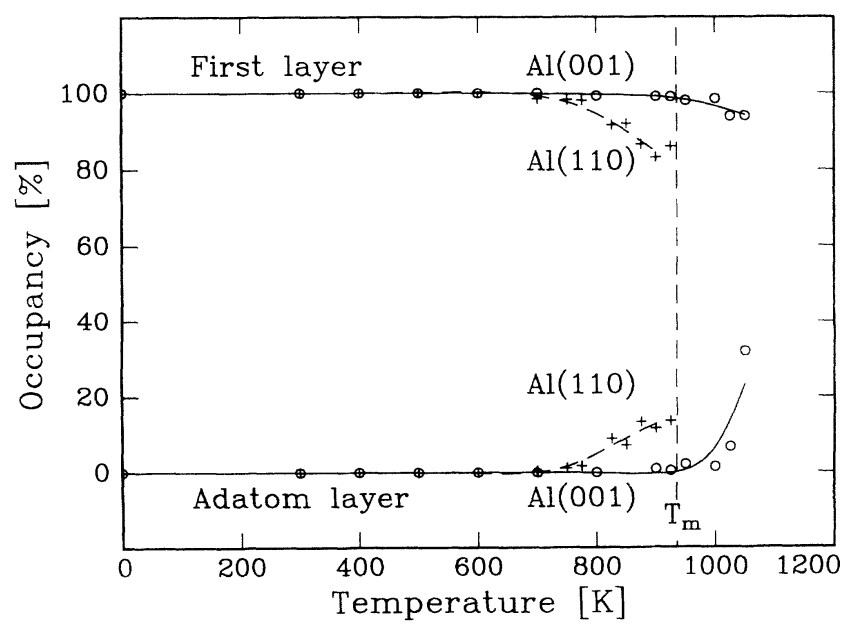

FIG. 6. Occupancies of the first layer and the adatom layer for $\mathrm{Al}(001)$ and $\mathrm{Al}(110)$. 
considered snapshot. The atom positions are denoted by $\mathbf{r}_{l}$. The in-plane reciprocal-lattice vector $\mathbf{k}$ has been chosen to probe nearest-neighbor distances along the $x=[110]$ direction:

$$
\mathbf{k}=\frac{2 \pi}{a(T)}(1,1,0) .
$$

Using $S_{j}(\mathbf{k})$ we have computed two layer-dependent order parameters. The first one is the average of the squared magnitude of $S_{j}(\mathbf{k}):\left\langle\left|S_{j}(\mathbf{k})\right|^{2}\right\rangle$; here \langle\rangle means averaging over MC cycles. This quantity probes the local thermal disorder within layer $j$ and is not sensitive to disorder in the interlayer registry.

The second order parameter is the square of the magnitude of the average of $S_{j}(\mathbf{k}):\left|\left\langle S_{j}(\mathbf{k})\right\rangle\right|^{2}$. This order parameter not only probes the in-plane disorder within layer $j$, but it also decreases when the entire layer is shifted away from perfect lateral registry with the bulk layers (the origin being defined with respect to the bulk). Such shifts can occur as a finite-size artifact of the simulation of a small periodically repeated unit cell. ${ }^{21}$ Both order parameters are equal to unity for a static fcc layer in perfect registry with the substrate, and approach $1 / N_{j}$ for a totally disordered or molten layer. By comparing these two order parameters, we can quantify the influence of the artificial shifting of layers parallel to the surface.

The shifting forms an intrinsic problem of MC simulations which make use of a finite cell and apply periodic boundary conditions. Once a layer of atoms has moved parallel to the surface, there is no direct restoring force in the same layer but only a weak interlayer force. The result is a low-frequency oscillation of the center of mass of the layer. MEIS is extremely sensitive to small relative shifts of layers of atoms. So a reliable comparison with ion-scattering measurements is only possible if we correct for the influence of the shifts. It is important to realize that most properties are not influenced noticeably by these shifts, because the amplitude of the oscillation is small and involves an extremely low energy. The difference in the center-of-mass position between the top and the second layer is at maximum $0.03 \AA$ at $T_{m}$. By relaxing unit cells with and without shifted layers, we have checked that the shifting of the layers does not influence the surface relaxation. The energy differences between shifted and nonshifted layers is quadratic in the size of the shift. If the number of atoms in a layer is doubled, the amplitude of the shifts goes down only by a factor $1 / \sqrt{2}$ at the expense of a large increase in calculation time. At $T_{m}$ the maximum energy difference involved in the shifting is about $60 \mathrm{meV}$ per layer. This is $O\left(k_{B} T\right)$ so also the phonon spectrum and the vibrations will not change in a significant way.

In Fig. $7, \ln \left\langle\left|S_{j}(\mathbf{k})\right|^{2}\right\rangle$ is shown as a function of temperature for the top four layers and a layer in the bulk of the crystal. If all layers would have vibrated harmonically (Debye-Waller model) all curves in Fig. 7 would have decreased linearly with temperature. The extra downward curvature, strongest for the surface, is caused by the anharmonicity of the vibrations. Above $\approx 400 \mathrm{~K}$ for the first layer and above $\approx 700 \mathrm{~K}$ for the fourth layer the

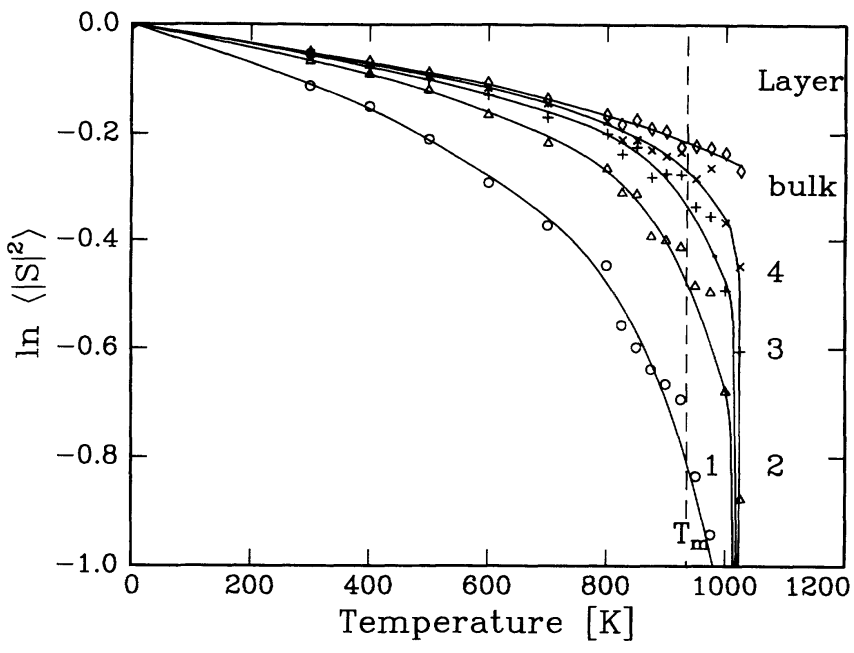

FIG. 7. $\ln \left\langle\left|S_{j}\right|^{2}\right\rangle$ as a function of temperature for layers $j=1,2,3,4$ and for bulk layers.

curves deviate from a straight line. At $T=1050 \mathrm{~K}$, $\ln \left\langle\left|S_{j}(\mathbf{k})\right|^{2}\right\rangle$ drops suddenly to $\approx \ln \left(\frac{1}{25}\right)=-3.22$, indicating a complete loss of order.

An easy way to quantify the layer shifts is to calculate the mean-square displacements $\sigma_{j}^{2}$ according to the two order parameters. Assuming nearly Gaussian vibrational displacement distributions, the layer-dependent vibration amplitudes are given by

$$
\begin{aligned}
& \sigma_{j}^{2}\left(\left\langle|S|^{2}\right\rangle\right)=-\frac{1}{|\mathbf{k}|^{2}} \ln \left[\frac{\left\langle\left|S_{j}(\mathbf{k})\right|^{2}\right\rangle-\left\langle 1 / N_{j}\right\rangle}{1-\left\langle 1 / N_{j}\right\rangle}\right], \\
& \sigma_{j}^{2}\left(|\langle S\rangle|^{2}\right)=-\frac{1}{|\mathbf{k}|^{2}} \ln \left[\frac{\left|\left\langle S_{j}(\mathbf{k})\right\rangle\right|^{2}-\left\langle 1 / N_{j}\right\rangle}{1-\left\langle 1 / N_{j}\right\rangle}\right] .
\end{aligned}
$$

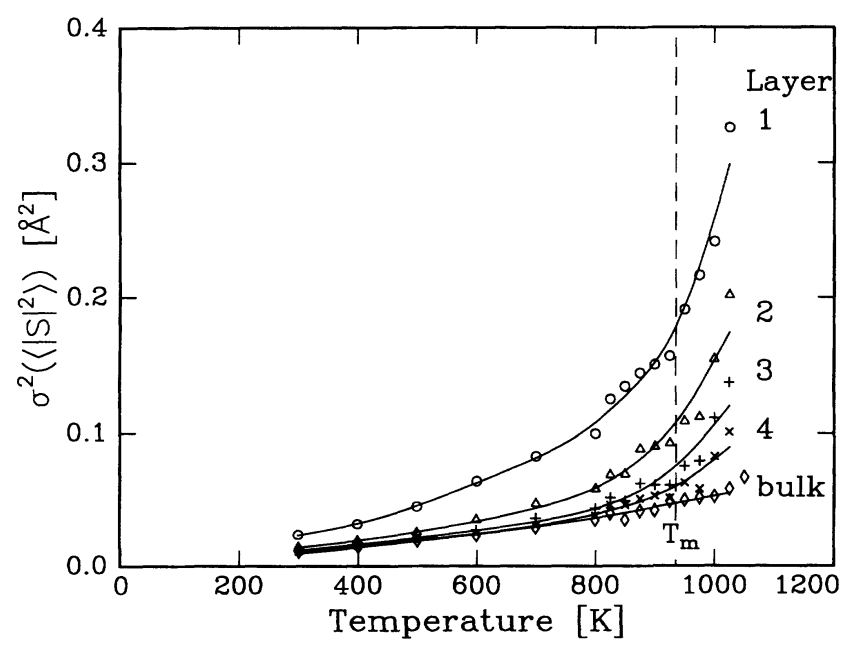

FIG. 8. Mean-square displacements in the $x=[110]$ direction, obtained from the average square of the structure factor, $\sigma_{j}^{2}\left(\left\langle|S|^{2}\right\rangle\right)$, as a function of $T$, for layers $j=1,2,3,4$ and for bulk layers. 


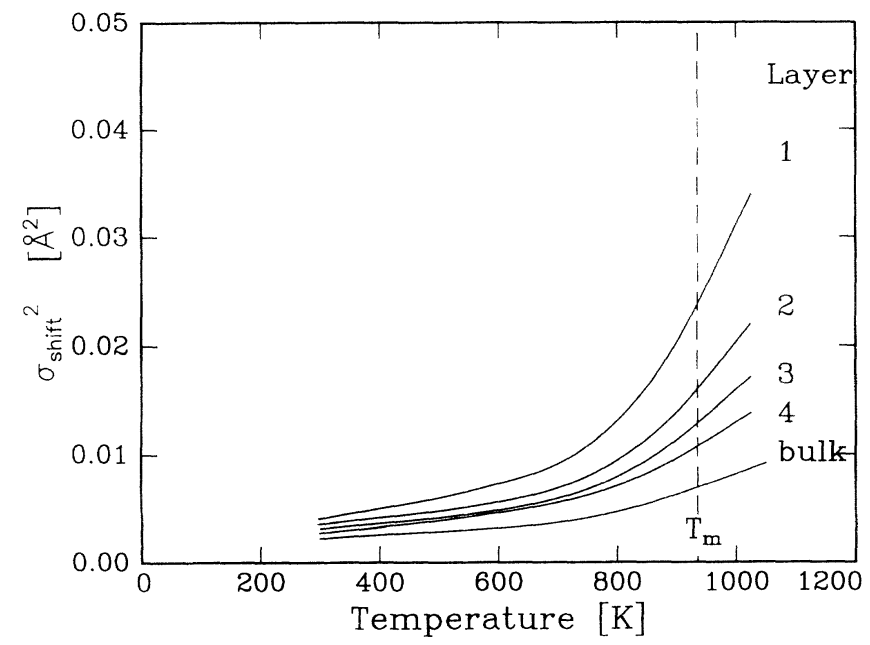

FIG. 9. Mean-square layer shifts $\sigma_{j \text {; shift }}^{2}$ for layers $j=1,2,3,4$ and for bulk layers.

The mean-square layer shift parallel to the surface

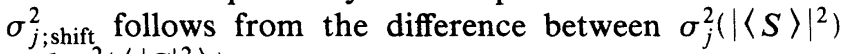
and $\sigma_{j}^{2}\left(\left\langle|S|^{2}\right\rangle\right)$.

In Fig. $8, \sigma_{j}^{2}\left(\left\langle|S|^{2}\right\rangle\right)$ is shown as a function of temperature and in Fig. 9 the mean-square layer shift $\sigma_{j \text {;shift }}^{2}$ is shown. For the widths $\sigma$ of the Gaussian curves in Fig. 4 we used $\sigma_{1}^{2}\left(|\langle S\rangle|^{2}\right)$. The mean-square layer shifts are one order of magnitude smaller than the mean-square vibrational displacements of the atoms in the layers.

The vibration amplitudes can also be calculated directly from the distributions of the positions of the atoms in a layer. Figures 10 and 11 show the mean-square displacements in the $x=[110]$ and $z=[001]$ directions. The parallel vibration amplitudes are larger than those perpendicular to the surface and the difference is largest for the first layer.

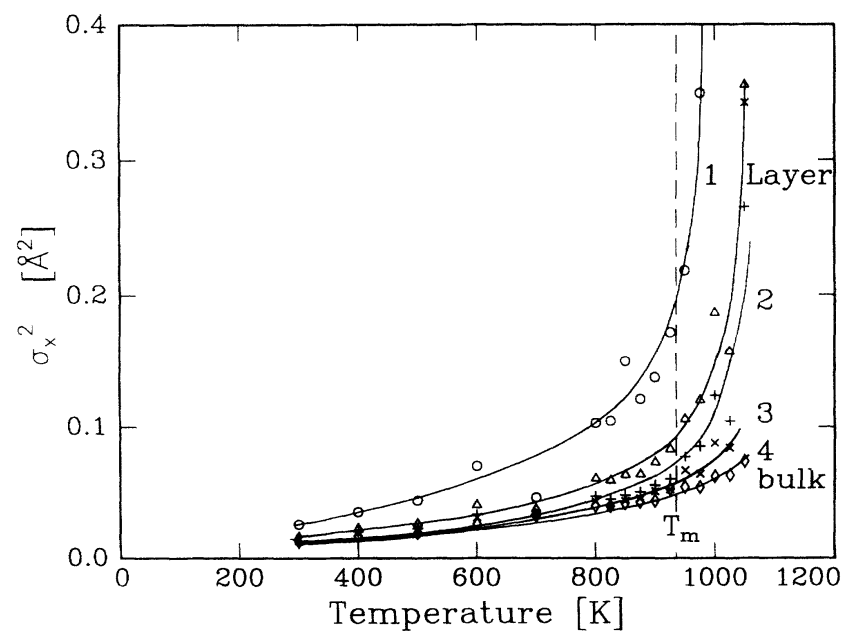

FIG. 10. Mean-square vibrational displacements $\sigma_{x}^{2}$ in the $x=[110]$ direction for layers $j=1,2,3,4$ and for bulk layers calculated by direct positional averaging.

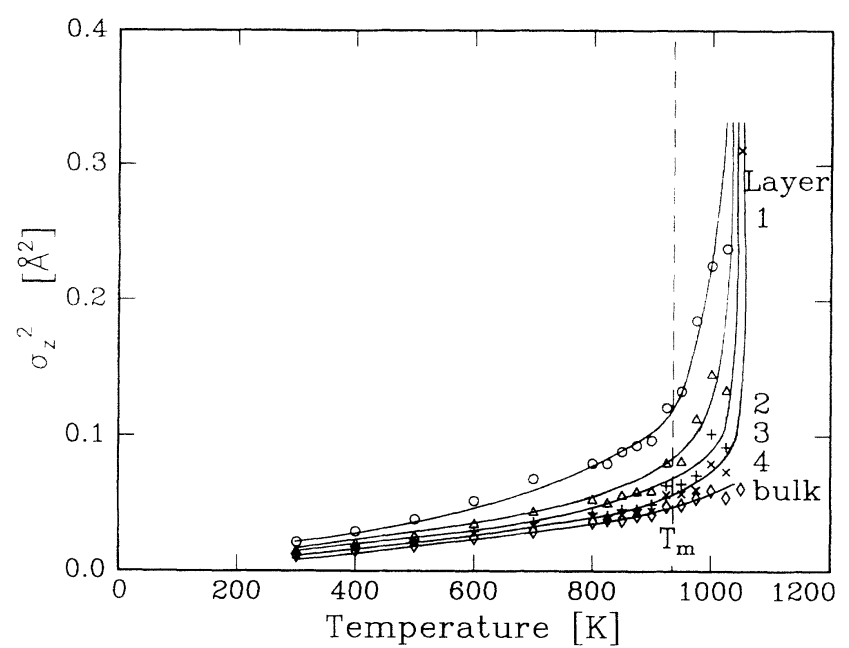

FIG. 11. Mean-square vibrational displacements $\sigma_{z}^{2}$ in the $z=[001]$ direction for layers $j=1,2,3,4$ and for bulk layers calculated by direct positional averaging.

\section{Ion-scattering calculations}

To study the sensitivity of the MEIS measurements to the anharmonicity of the vibrations, we computed ionscattering yields for the geometry of Fig. 1. First of all the ion-scattering yields were calculated for a collection of uncorrelated snapshots generated by the EMT simulation, after the unit cell was equilibrated. Typically 50 snapshots were used with 100-MC cycles in between (to make sure that the snapshots were independent). For each of these surfaces, the trajectories were calculated for $1000-\mathrm{H}^{+}$ions $/ \AA^{2}$ of $100 \mathrm{keV}$, impinging along the [011] direction and the same number along the outgoing [011] direction. In order to calculate the nuclear encounter probability of the ions in a computationally efficient way, ${ }^{3}$ the positions of the atoms in a unit cell were slightly spread out by an added small isotropic Gaussian probability density distribution around their positions in the snapshots. If the Gaussian width is chosen small enough (e.g., $\delta=0.05 \AA$ ), it does not affect the value of the backscattered yield. ${ }^{21}$ The resulting scattering intensities are shown by the crosses in Figs. 1 and 12.

Next, four "conventional" ion-scattering calculations ${ }^{48}$ were performed for the following configurations.

(a) A surface with bulk vibration amplitudes from Fig. 8.

(b) A surface with vibration amplitudes determined by a harmonic extrapolation in temperature from the vibration amplitudes at $300 \mathrm{~K}$. The root mean-square displacement in layer $j$ at temperature $T$ is then given by

$$
\sigma_{j}(T)=\frac{\sigma_{j}(300 \mathrm{~K})}{\sigma_{\text {bulk }}(300 \mathrm{~K})} \times \sigma_{\text {bulk }}(T) .
$$

(c) A surface with vibration amplitudes from $\sigma_{j}^{2}\left(\left\langle|S|^{2}\right\rangle\right)$ [Eq. (7)].

(d) A surface with vibration amplitudes from $\sigma_{j}^{2}\left(|\langle S\rangle|^{2}\right)$ [Eq. (8)]. 


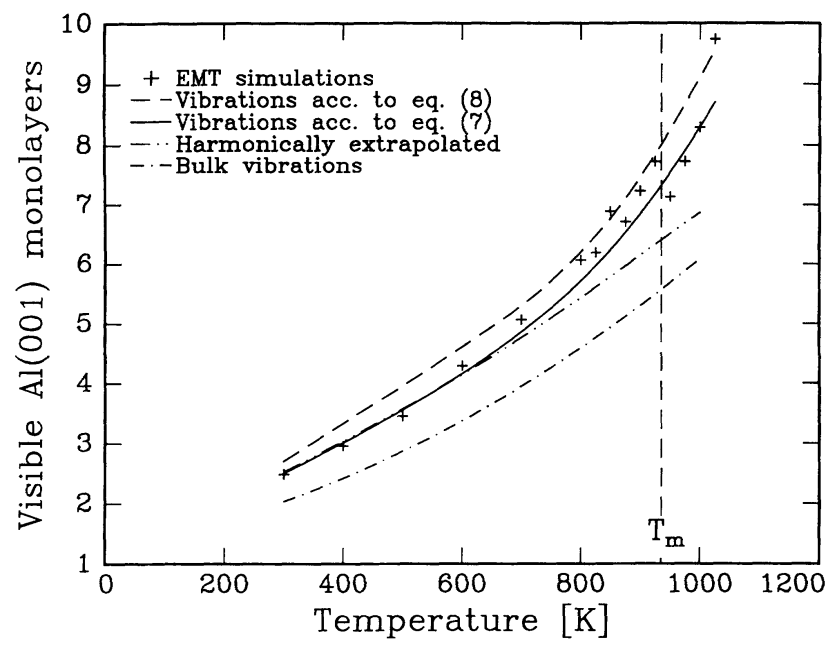

FIG. 12. Number of visible $\mathrm{Al}(001)$ monolayers for $100-\mathrm{keV}$ $\mathrm{H}^{+}$with incoming direction [011] and outgoing direction [011], calculated from EMT-simulation snapshots $(+)$, and calculated for Gaussian displacement distributions with harmonically increasing (a),(b) and anharmonically increasing (c),(d) meansquare displacements (see text).

These ion-scattering calculations were performed with $5000-\mathrm{H}^{+}$ions $/ \AA^{2}$ of $100 \mathrm{keV}$ again impinging along the $[01 \overline{1}]$ direction and emerging along [011]. All four surfaces were relaxed according to Fig. 5. The vibrational displacements were assumed to be Gaussian, uncorrelated, and isotropic. The last assumption is valid for the $x$ and $y$ directions parallel to the surface because of symmetry and almost valid perpendicular to the surface.

Figure 12 shows the backscattered yields for these four vibrational configurations together with the EMT result. Comparing the EMT results with those for the crystal with bulk vibration amplitudes, configuration (a), we see an increase in backscattering yield of 0.6 monolayer at room temperature and 1.7 monolayers at the bulk melting point, due to the enhanced surface vibrations. In Fig. 7 it was shown that at room temperature the crystal vibrates almost harmonically. If the vibration amplitudes are extrapolated harmonically, by fixing the ratios of surface to bulk vibration amplitudes to their roomtemperature values, configuration (b) [Eq. (9)], the extra ion-scattering yield with respect to the bulk crystal is almost a constant (0.7-0.8 monolayer).

The effect of the anharmonicity on the ionbackscattering yield is demonstrated by the two upper curves, (c) and (d), in Fig. 12. Again the displacement distributions were chosen Gaussian but the amplitudes had been adapted to fit the EMT results. In this way we tested the idea that it is the width, rather than the precise shape of the distributions, that the ion scattering is most sensitive to. The calculations with these vibration amplitudes agree very well with the EMT simulations. The ion-scattering yield of the crystal with vibration amplitudes from $\sigma_{j}\left(\left|\langle S\rangle^{2}\right|\right)$, configuration (d) is about 0.4 monolayer higher than the one calculated by means of $\sigma_{j}\left(\left\langle|S|^{2}\right\rangle\right)$, configuration (c). As discussed, the difference is caused by the artificial shifting of the layers parallel to the surface.

\section{DISCUSSION}

The $\mathrm{Al}(001)$ surface has a density of $1.22 \times 10^{15}$ atoms $/ \mathrm{cm}^{2}$, between that of the close-packed $\mathrm{Al}(111)$ and the open $\mathrm{Al}(110)$ surfaces. This sequence in atomic densities at the surface is also reflected in the solid-vapor interfacial free energies [ $\mathrm{Al}(111)$ has the lowest $\gamma_{\mathrm{sv}}$ and $\mathrm{Al}(110)$ the highest] and thus in the melting behavior. Although $\gamma_{\text {sv }}$ has been calculated for the $\mathrm{Al}(001)$ surface [0.830 (Ref. 49), 1.081 (Ref. 63), 0.370 (Ref. 60), 1.230 (Ref. 61) $\mathrm{Jm}^{-2}$ ] and $\gamma_{\mathrm{lv}}$ has been determined experimentally $\left[0.864 \mathrm{Jm}^{-2}\right.$ (Ref. 43)] there is no reliable value for $\gamma_{\mathrm{sl}}$ close to the bulk melting point. So, as already has been stated, it is not possible to predict the surface stability of $\mathrm{Al}(001)$ from the difference in surface free energies.

In our MC simulations we filtered out the shifting of layers parallel to the surface caused by the finite size of the unit cell. This has been done by averaging the structure factor in the proper way. The amplitude of the shifting depends strongly on the packing density of atoms in a layer: the $\mathrm{Al}(111)$ surface exhibits the strongest shifting and on the $\mathrm{Al}(110)$ there is almost no shifting. For $\mathrm{Al}(001)$ the influence of layer shifts on the ion-scattering intensity is rather small.

The anharmonicity of the surface is manifest in several properties: the surface enhancement of the thermalexpansion coefficient, the development of non-Gaussian tails in the atomic position distributions at the surface, and the temperature-dependent increase in the surfaceto-bulk ratio of the vibration amplitudes.

The Al surface is more stable close to the bulk melting point than other fcc(001) surfaces, such as $\mathrm{Au}(001), 8,9$ $\mathrm{Cu}(001),{ }^{26,27}$ and $\mathrm{Pb}(001)$ (Refs. 29, 35, and 36). The density of adatom/vacancy pairs close to the melting point is an order of magnitude less on the $\mathrm{Al}(001)$ surface than on $\mathrm{Cu}(001)$. Nevertheless, the anharmonicity on the $\mathrm{Al}(001)$ surface is larger than on $\mathrm{Cu}(001)$, as can be inferred from the ratios of surface to bulk vibration amplitudes in our simulations and those in Ref. 27. Experimental evidence for anharmonicity on $\mathrm{Cu}(001)$ has been presented by $\mathrm{He}$ atom scattering. ${ }^{65}$

Comparing the vibrational distributions of $\mathrm{Al}(001)$ to the ones of the $\mathrm{Al}(110)$ surface, we see similar anharmonicity at low temperatures, up to the temperature where the disordering at the Al(110) surface starts (about 750 $\mathrm{K})$. The vibration amplitudes for the first atomic layer of both surfaces increase, along the [110] direction, from $0.16 \AA$ at room temperature to $0.24 \AA$ at $700 \mathrm{~K}$. The observation that the (110) surface melts, whereas the (001) surface remains stable up to temperatures where the anharmonicity is even much stronger than at $700 \mathrm{~K}$, suggests that the anharmonicity is not making these surfaces vibrationally unstable. The mechanism by which the first layer of a melting surface becomes disordered seems to be completely dominated by the presence of adatoms and vacancies (Fig. 6).

\section{CONCLUSIONS}

Using MEIS experiments and MC simulations we have demonstrated that the $\mathrm{Al}(001)$ surface stays well ordered 
up to the bulk melting point. No surface melting has been observed, but the surface atoms vibrate strongly anharmonically. The simulations indicate that the difference in thermal-expansion coefficient, from room temperature to the bulk melting point, of the first layer of $\mathrm{Al}(001)$ is enhanced by a factor 2.5 over the bulk value. The high-temperature behavior of $\mathrm{Al}(001)$ is different from that of the $\mathrm{Au}(001), \mathrm{Cu}(001)$, and $\mathrm{Pb}(001)$ surfaces, which all seem to develop a finite amount of disorder. The ion-scattering measurements and calculations show that MEIS is sensitive to the anharmonicity of the vibrations. The effective-medium potential describes the temperature-dependent behavior of the $\mathrm{Al}(001)$ surface excellently, without any adjustable parameter.

\section{ACKNOWLEDGMENTS}

The authors thank R. I. J. M. Koper for the preparation of the $\mathrm{Al}(001)$ sample and $\mathrm{D}$. Frenkel for valuable discussions. This work is part of the research program of the Stichting voor Fundamenteel Onderzoek der Materie (FOM) and was made possible by financial support from the Nederlandse Organisatie voor Wetenschappelijk Onderzoek (NWO).
${ }^{1}$ I. K. Robinson, E. Vlieg, H. Hornis, and E. H. Conrad, Phys. Rev. Lett. 67, 1890 (1991).

${ }^{2}$ M. den Nijs, Phys. Rev. B 46, 10386 (1992).

3J. W. M. Frenken, P. M. J. Marée, and J. F. Van der Veen, Phys. Rev. B 34, 7506 (1986).

${ }^{4}$ J. F. Van der Veen and J. W. M. Frenken, Surf. Sci. 251/252, 1 (1991).

${ }^{5}$ G. Bilalbegović, F. Ercolessi, and E. Tosatti, Europhys. Lett. 17, 333 (1992).

${ }^{6}$ H. M. Van Pinxteren and J. W. M. Frenken, Europhys. Lett. 21, 43 (1993).

${ }^{7}$ R. J. Phaneuf and E. D. Williams, Phys. Rev. Lett. 58, 2563 (1987).

${ }^{8}$ S. G. J. Mochrie, D. M. Zehner, B. M. Ocko, and D. Gibbs, Phys. Rev. Lett. 64, 2925 (1990).

${ }^{9}$ B. M. Ocko, D. Gibbs, K. G. Huang, D. M. Zehner, and S. G. J. Mochrie, Phys. Rev. B 44, 6429 (1991).

${ }^{10}$ R. Lipowsky and W. Speth, Phys. Rev. B 28, 3983 (1983).

${ }^{11}$ A. Trayanov and E. Tosatti, Phys. Rev. Lett. 87, 2207 (1987).

12J. G. Dash, Contemp. Phys. 30, 89 (1989).

${ }^{13}$ J. F. Van der Veen, Phase Transitions in Surface Films 2, edited by H. Taub (Plenum, New York, 1991).

${ }^{14}$ C. S. Jayanthi, E. Tosatti, and L. Pietronero, Phys. Rev. B 31, 3456 (1985).

${ }^{15}$ F. F. Abraham and J. Q. Broughton, Phys. Rev. Lett. 56, 734 (1986).

${ }^{16}$ P. Stoltze, J. K. Nørskov, and U. Landman, Surf. Sci. Lett. 220, L693 (1989).

${ }^{17}$ M. Karimi and M. Mostoller, Phys. Rev. B 45, 6289 (1992).

${ }^{18} \mathrm{P}$. von Blanckenhagen, W. Schommers, and V. Voegele, J. Vac. Sci. Technol. A 5, 649 (1987).

${ }^{19}$ P. Stoltze, J. K. Nørskov, and U. Landman, Phys. Rev. Lett. 61, 440 (1988).

${ }^{20}$ A. W. Denier van der Gon, R. J. Smith, J. M. Gay, D. J. O'Connor, and J. F. Van der Veen, Surf. Sci. 227, 143 (1990).

${ }^{21}$ A. W. Denier van der Gon, D. Frenkel, J. W. M. Frenken, R. J. Smith, and P. Stoltze, Surf. Sci. 256, 385 (1991).

${ }^{22}$ H. Dosch, T. Höfer, J. Peisl, and R. L. Johnson, Europhys. Lett. 15, 527 (1991).

${ }^{23}$ P. Carnevali, F. Ercolessi, and E. Tosatti, Phys. Rev. B 36, 6701 (1987)

${ }^{24}$ F. Ercolessi, S. Iarlori, O. Tomagnini, E. Tosatti, and X. J. Chen, Surf. Sci. 251/252, 645 (1991).

${ }^{25}$ A. Hoss, M. Nold, P. von Blanckenhagen, and O. Meyer, Phys. Rev. B 45, 8714 (1992).

${ }^{26}$ K. D. Stock, Surf. Sci. 91, 655 (1980).

${ }^{27}$ H. Häkkinen and M. Manninen, Phys. Rev. B 46, 1725 (1992).

${ }^{28}$ K. C. Prince, U. Breuer, and H. P. Bonzel, Phys. Rev. Lett.
60, 1146 (1988).

${ }^{29}$ B. Pluis, A. W. Denier van der Gon, J. F. Van der Veen, and A. J. Riemersma, Surf. Sci. 239, 265 (1990).

${ }^{30}$ S. Speller, M. Schleberger, and W. Heiland, Surf. Sci. 269/270, 229 (1992).

${ }^{31}$ H. P. Bonzel, U. Breuer, B. Voigtländer, and E. Zeldov, Surf. Sci. 272, 10 (1992).

${ }^{32}$ B. Pluis, D. Frenkel, and J. F. Van der Veen, Surf. Sci. 239, 282 (1990).

${ }^{33}$ A. A. Chernov and L. V. Mikheev, Phys. Rev. Lett. 60, 2488 (1988).

${ }^{34}$ G. Bilalbegović, F. Ercolessi, and E. Tosatti, Surf. Sci. Lett. 258, L676 (1991).

${ }^{35}$ H. M. van Pinxteren and J. W. M. Frenken, Surf. Sci. 275, 383 (1992).

${ }^{36}$ H.-N. Yang, K. Fang, G.-C. Wang, and T.-M. Lu, Phys. Rev. B 44, 1306 (1991).

${ }^{37}$ P. Thiry, G. Jezequel, and Y. Petroff, J. Vac. Sci. Technol. A 5, 892 (1987).

${ }^{38}$ G. Jezequel, P. Thiry, G. Rossi, K. Hricovini, and Y. Petroff, Surf. Sci. 189/190, 605 (1987).

${ }^{39}$ J. F. Van der Veen, B. Pluis, and A. W. Denier van der Gon, Chemistry and Physics of Solid Surfaces VII, edited by R. Vanselow and R. F. Howe (Springer, Berlin, 1988).

${ }^{40}$ W. C. Turkenburg, E. De Haas, A. F. Neuteboom, J. Ladru, and H. H. Kersten, Nucl. Instrum. Methods 126, 241 (1975).

${ }^{41}$ R. G. Smeenk, R. M. Tromp, H. H. Kersten, A. J. H. Boerboom, and F. W. Saris, Nucl. Instrum. Methods Phys. Res. 195, 581 (1982).

${ }^{42}$ P. M. J. Mareé, A. P. De Jongh, J. W. Derks, and J. F. Van der Veen, Nucl. Instrum. Methods Phys. Res. B 28, 76 (1987).

${ }^{43}$ Handbook of Chemistry and Physics, 70th edition, edited by R. C. Weast (CRC Press, Florida, 1989).

${ }^{44} \mathrm{G}$. Lewis, Fundamentals of Vacuum Science and Technology (McGraw-Hill, New York, 1965).

${ }^{45}$ P. M. Zagwijn, A. M. Molenbroek, J. Vrijmoeth, G. J. Ruwiel, R. M. Uiterlinden, J. ter Horst, J. ter Beek, and J. W. M. Frenken, Nucl. Instrum. Methods Phys. Res. B (to be published).

46J. F. Van der Veen, Surf. Sci. Rep. 5, 199 (1985).

${ }^{47}$ J. F. Van der Veen, B. Pluis, and A. W. Denier van der Gon, in Kinetics of Ordering and Growth at Surfaces, edited by $\mathrm{M}$. G. Lagally (Plenum, New York, 1990).

${ }^{48} \mathrm{~J}$. W. M. Frenken, R. M. Tromp, and J. F. Van der Veen, Nucl. Instrum. Methods Phys. Res. B 17, 334 (1986).

${ }^{49}$ K. W. Jacobsen, J. K. Nørskov, and M. J. Puska, Phys. Rev. B 35, 7423 (1987).

${ }^{50}$ N. Metropolis, A. W. Rosenbluth, M. N. Rosenbluth, A. H. 
Teller, and E. Teller, J. Chem. Phys. 21, 1087 (1953).

${ }^{51}$ The expression for $a(T)$ in Ref. 21 had the coefficients in front of the $T$ and $T^{2}$ wrong by a factor 10 . However, the bulk lattice constants used in the calculations of Ref. 21 were correct.

${ }^{52} \mathrm{G}$. Simmons and H. Wang, Single Crystal Elastic Constants and Calculated Aggregate Properties: A Handbook (MIT Press, Cambridge, 1971).

${ }^{53}$ C. Kittel, Introduction to Solid State Physics (Wiley, New York, 1968).

${ }^{54}$ In the case of $\mathrm{Al}(110)$ (Ref. 21) the simulations were also compared with the experiments by using an absolute temperature scale. Up to about $850 \mathrm{~K}$, where no liquid is present on this surface, the use of the absolute scale is correct. Close to $T_{m}$ a temperature scale relative to $T_{m}$ should be used. The difference between the experimental and simulated melting points does not affect the conclusions of Ref. 21 because the highest temperature range was left unexplored.

${ }^{55}$ D.W. Jepsen, P. M. Marcus, and F. Jona, Phys. Rev. B 5, 3933 (1972).
${ }^{56}$ F. Jona, D. Sondericker, and P. M. Marcus, J. Phys. C 13, L155 (1980).

${ }^{57}$ A. Bianconi and R. Z. Bachrach, Phys. Rev. Lett. 42, 104 (1979).

${ }^{58}$ N. Masud, R. Baudoing, D. Aberdam, and C. Gaubert, Surf. Sci. 133, 580 (1983).

${ }^{59}$ The small difference between the first-layer relaxations calculated in this work and in Ref. 49 stems from a difference in the cutoff radius of the interactions.

${ }^{60}$ Ning Ting, Yu Qingliang, and Ye Yiying, Surf. Sci. 206, L857 (1988).

${ }^{61}$ T. J. Raeker and A. E. DePristo, Phys. Rev. B 39, 9967 (1989).

${ }^{62}$ R. N. Barnett, U. Landman, and C. L. Cleveland, Phys. Rev. B 28, 1685 (1983).

${ }^{63}$ K.-P. Bohnen and K.-M. Ho, Surf. Sci. 207, 105 (1988).

${ }^{64}$ S. Debiaggi and A. Caro, J. Phys. Condens. Matter 4, 3905 (1992).

${ }^{65}$ G. Armand, D. Gorse, J. Lapujoulade, and J. R. Manson, Europhys. Lett. 3, 1113 (1987). 


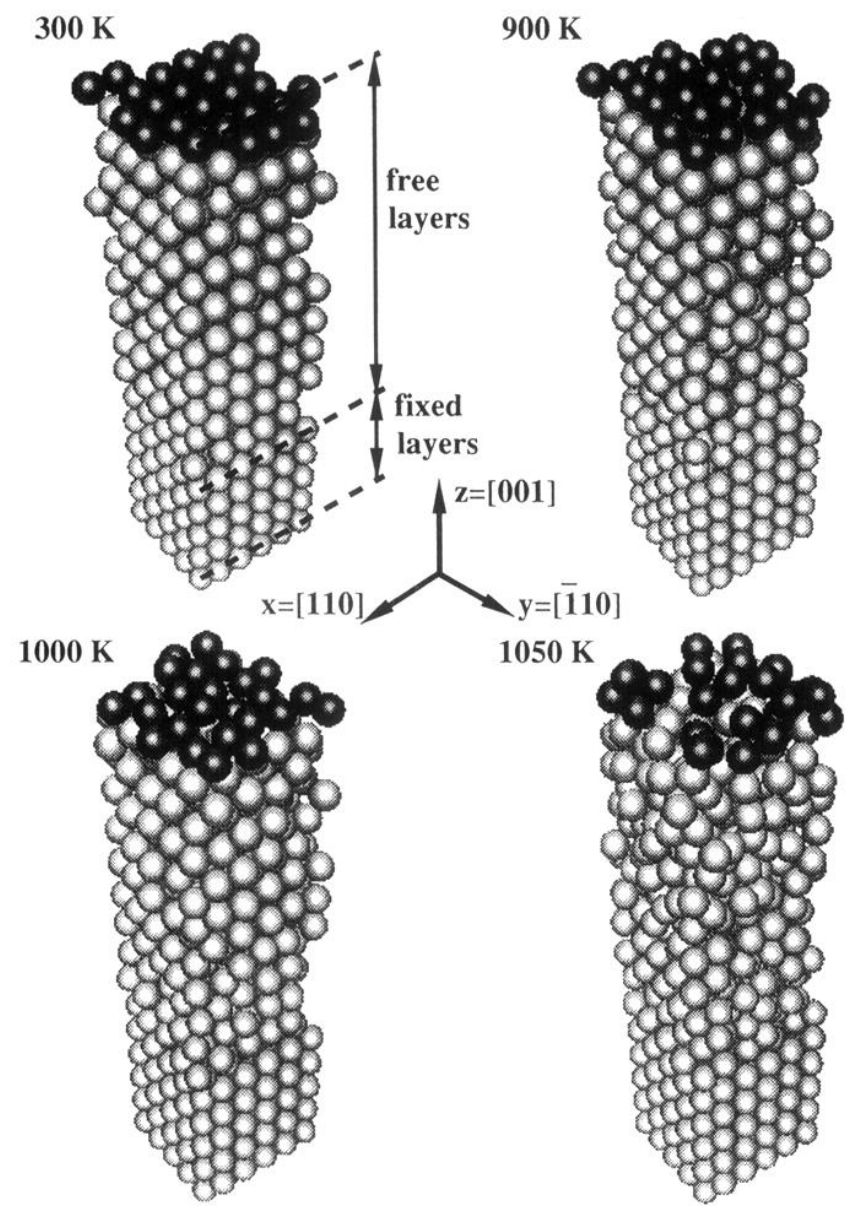

FIG. 3. Perspective view of snapshots of the unit cell at $T=300,900,1000$, and $1050 \mathrm{~K}$. The first-layer atoms have been shaded darker. 